\section{IAP Immunization Timetable 2012 Clarifications}

We read the recent IAPCOI's Consensus Recommendations on Immunization and IAP Immunization timetable 2012 [1]. We appreciate the sincerity and efforts put by IAPCOI in formulating these guidelines. Before we accept these guidelines and bring them into clinical practice, we would like to have few clarifications and share possible technical difficulties:

1. Omitting OPV from routine schedule at 6,10 and 14 weeks. This is likely to create confusion among public, when one group is advised to take OPV and other group for not using OPV in routine schedule, purely on the basis of economic background. This is also likely to increase the demand of IPV, for which public sector may not be prepared yet. Wouldn't it have been wise to prepare such recommendation; while enforcing similar changes in National schedule too, when Government is prepared with enough stocks of IPV. So that confusion in public, at this vital stage of polio eradication could have been avoided.

2. When one decides to use IPV and not OPV in routine schedule, we are not convinced about using IPV-OPV schedule. When IPV is proved to be highly efficacious and able to provide equal mucosal immunity, why not go for only IPV schedule $[2,3]$ ? What is the justification for advising OPV later at 6 months and 9 months, knowing the difficulty in getting people at 6 months?

3. Regarding rotavirus vaccine, in absence of any efficacy trial on this issue from India, poor immunogenicity shown in developing countries, and prevalent strains not covered by presently available vaccines [4]; how justified are we in recommending this for routine use?

4. Regarding boosters of MMR and varicella, we would like to know the justification for recommending it at five years. Before recommending such boosters, we should know the status of persistence of protective antibody titres against these diseases at later ages after primary vaccination in our children. When natural infections are still likely to play a significant role in boosting immunity in our children, even if we need boosters, probably MMR and varicella boosters at 10 years would provide more robust immune response in our children rather than then giving at 5 years [5].

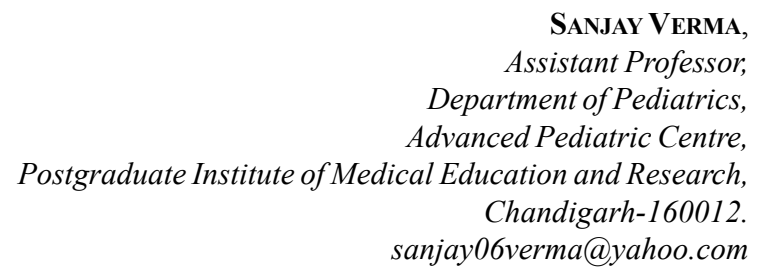

\section{REFERENCES}

1. IAPCOI. Consensus Recommendations on Immunization and IAP Immunization Timetable 2012. Indian Pediatr. 2012;49:549-64.

2. Krishnan R, Jadhav M, John TJ. Efficacy of inactivated poliovirus vaccine in India. Bull World Health Organ. 1983;61:689-92.

3. Selvakumar R, John TJ. Intestinal immunity induced by inactivated poliovirus vaccine. Vaccine. 1987;5:141-4.

4. Lodha R, Shah D. Prevention of rotavirus diarrhea in India: Is vaccination the only stretegy? Indian Pediatr. 2012;49:441-4.

5. Deshmukh V. MMR Vaccination: New thoughts. Indian Pediatr. 2006;43: 555.

\section{REPLY}

1. Again, we should not confuse with the committee's recommendations which are mainly for office practice. Considering the current state of polio eradication in the country, the committee believes that persisting with OPV poses significant risks both at the individual and public segment, vaccine associated paralytic polio (VAPP) at the former and circulating vaccine derived poliomyelitis (cVDPVs) at the latter. The move will also provide a timely policy 'signal' to Indian policymakers to expedite consultations on endgame and post-eradication vaccine policy. The recent SAGE April 2012 Working Group meeting confirmed early universal IPV introduction (as early as October 2013) integrated into routine immunization program (before planned April 2014 tOPV to bOPV switch) of the country [1]. So, even at the public sector, there is great pressure to introduce IPV to facilitate gradual albeit staggered OPV removal from routine immunization.

2. It is indeed a daunting task of how to strike a balance between individual and public sector use while formulating any recommendation on polio vaccines considering the sensitive nature of the polio eradication program in the country. Since OPV is still in use in the country and SIAs are still organized, we have decided to move gradually, hence the sequential schedule. This schedule will meet our objectives of providing immunity against VAPP and cVDPV, and at the same time permits the benefits of OPV. Even WHO has instructed to move from sequential than to all IPV schedule for countries 